\title{
PREVALÊNCIA DE CÁRIE ASSOCIADA À INFLUÊNCIA DA PROPAGANDA NOS HÁBITOS ALIMENTARES DE ESCOLARES
}

\section{CARIES PREVALENCE IN ASSOCIATION WITH ADVERTISEMENT INFLUENCE ON THE FOOD HABITS OF STUDENTS}

\author{
Regina de Nazaré Marreiros Tavares* \\ Ana Lucia Herdy Teixeira* \\ Lizete Lopes da Costa"** \\ Lisiana Carla Tenório Amorim $* *$ \\ Lilian Coranni Macaferri Licatti ${ }^{\cdots \cdots * *}$ \\ Luciane Zanin $* * * * *$ \\ Flávia Martão Flório ${ }^{* \ldots+\cdots * \cdots}$

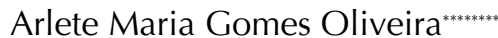

\begin{abstract}
RESUMO
O objetivo do estudo foi avaliar a influência das propagandas da TV nos hábitos alimentares de escolares de 12 anos e associação com a prevalência de cárie. Estudo epidemiológico observacional transversal em 294 estudantes utilizou Índice CPO e Questionário estruturado. Os resultados mostraram que quem assistia SBT e TV das 9 às 12 horas tinha 1,73 vezes e 1,66 vezes mais chance de ter cárie. A correlação foi positiva para o tempo de exposição à TV e prevalência de cárie $(p=0,02)$. Concluiu-se que o tempo de exposição à TV pode influenciar hábitos alimentares em crianças e adolescentes, contribuindo para o desenvolvimento da cárie dental.

DESCRITORES: Propaganda • Hábitos alimentares • Cárie dentária
\end{abstract}

\section{ABSTRACT}

The aim of this study was to assess the influence of TV' propaganda on food habits of students aged 12 years and its association with the prevalence of caries. A cross sectional observational epidemiological study of 294 students used DMFT and structured questionnaire. The outcomes showed that who watched SBT and TV from 9 to $12 \mathrm{o}^{\prime}$ clock had 1.73 times and 1.66 times more likely to have caries. The correlation was positive to the time for watching TV and prevalence of caries $(p=0.02)$. It was concluded that the time for watching TV can influence the food habits of children and adolescents, contributing to the development of dental caries.

DESCRIPTORS: Propaganda • Food habits • Dental caries

* Mestre Odontologia -Saúde Coletiva - Faculdade São Leopoldo Mandic - Campinas - SP. E-mail : rtavares13@hotmail.com

** Mestranda Odontologia - Saúde Coletiva - Faculdade São Leopoldo Mandic - Campinas - SP. E-mail : analuciaherdy@gmail.com

*** Mestre Odontologia - Saúde Coletiva - Faculdade São Leopoldo Mandic - Campinas - SP. E-mail : lopeslizete@yahoo.com.br

**** Mestre Odontologia -Saúde Coletiva - Prefeitura Municipal de Maceió - Alagoas - Brasil. E-mail: lisiana@globo.com

***** Mestre Odontologia -Saúde Coletiva - Serviço nacional de aprendizagem comercial - SENAC - SP. E-mail: lilian.mlicatti@sp.senac.b

****** Doutorado Odontologia (Cariologia) - Professora Faculdade São Leopoldo Mandic - Campinas - SP. E-mail: zaninsouza@yahoo.com.br

******* Doutorado Odontologia (Cariologia) - Professora Faculdade São Leopoldo Mandic - Campinas - SP. E-mail: flavia.florio@slmandic.edu.br

******** Doutora Odontologia (Saúde Coletiva) - Professora Faculdade São Leopoldo Mandic - Campinas - SP. Email: arlete.oliveira@slmandic.edu.br 


\section{N T R O D UÇÃ O}

A associação entre o consumo de sacarose e a prevalência de cárie é bem estabelecida ${ }^{1,2}$, e a fase escolar é um excelente momento para o incentivo da formação de hábitos alimentares saudáveis e de saúde oral de crianças e adolescentes. Com o advento da televisão, a propaganda de produtos alimentícios e sua qualidade nutricional apresentam-se de forma inadequada ao consumo infantil, uma vez que possuem quantidades inapropriadas de gorduras, açúcares e sal, contribuindo, assim, para o aumento da prevalência de doenças crônicas como obesidade, diabetes, doenças cardiovasculares e cárie dental ${ }^{3}$.

A alusão à propaganda de alimentos encontra-se à luz das discussões entre profissionais da área de saúde, uma vez que existem fortes evidências de que a mídia televisiva tão presente na atual sociedade capitalista, em que o consumo é muito estimulado, vem exercendo papel importante na mudança dos padrões alimentares da população, através da exposição às peças publicitárias de produtos alimentícios, principalmente dos industrializados, fast foods, dentre outros ${ }^{4}$. A disseminação do consumo desse tipo de alimento considerado "junk food" (lixo alimentar) é maior no público infantil, provavelmente em decorrência da falta de opções saudáveis na escola e em casa, além das frequentes publicidades de alimentos que influenciam de forma negativa as escolhas alimentares da família ${ }^{4-5}$. Os pais, muitas vezes, têm consciência de que alimentos industrializados não são saudáveis, porém, em função do tempo laboral diário, são influenciados pela facilidade na preparação desses alimentos para o consumo ${ }^{6}$.

A indústria realça o sabor dos alimentos processados através do alto teor de açúcares e gorduras, considerando o atrativo do paladar doce e gorduroso, promovendo a associação com personagens que fazem parte de seu imaginário social criando uma relação de confiança e afetividade com o produto, dois elementos que são essenciais na infância ${ }^{7,8}$. Dessa forma, o público infantil se torna vulnerável a essas mensagens de marketing emo- cional, inclusive àquelas que encorajam a convencer os pais a respeito de compras ${ }^{9}$; por isso a regulamentação da propaganda de certos produtos poderia estimular escolhas mais saudáveis na alimentação, possibilitando melhor saúde geral ${ }^{10}$.

A TV é o veículo preferido da publicidade pelo grande alcance de público, porém são as crianças que costumam interagir com a "telinha" por período maior de tempo ao longo do dia ${ }^{11}$; por isso o tempo de exposição à televisão pode ser fator de risco para o desenvolvimento de doenças. Estudo tem demonstrado que quanto maior o tempo em que as crianças permanecem diante da TV, maior é o risco de consumo de alimentos contendo açúcares, favorecendo a aquisição da cárie dental, observação esta complementada após análise do conteúdo dos comerciais pelos autores, da ausência de publicidade de alimentos saudáveis como frutas, legumes e verduras ${ }^{12}$.

A maioria das propagandas relacionadas a alimentos veiculadas nos canais abertos, destina-se ao público infantil, apresentando baixos valores nutritivos, altos valores calóricos e, de forma apelativa, utilizando artifícios visuais como cores, uso de desenhos e brindes de personagens infantis. Não se observa nenhuma propaganda incentivando ao consumo de frutas e legumes ${ }^{9}$.

De acordo com Fechine et al. ${ }^{6}$ (2015), por mais que haja a observância por parte dos responsáveis, de que o consumo de alimentos industrializados não seja benéfico e saudável às crianças, é comum a oferta diária desses alimentos em casa, apontada como uma prática instituída a partir de modelos concebidos pela sociedade moderna, evento observado com maior predominância em populações menos favorecidas economicamente. Em sua pesquisa, os autores concluíram que, mesmo havendo a oferta da merenda escolar, existem casos de comercialização de alimentos industrializados e guloseimas na porta das creches, mesmo na zona rural. Esse tipo de serviço acaba por impedir os esforços da escola em oferecer uma alimentação mais adequada e saudável para as crianças, necessitando de medidas regulatórias por parte da Secretaria de Edu-
TAVARES RNM

TEIXEIRA ALH

COSTA LL

AMORIM LCT

LicATTI LCM

ZANIN L

FLORIO FM

OLIVEIRA AMG

PREVALENCIA DE

CÁRIE ASSOCIADA

À INFLUÊNCIA

DA PROPAGANDA

NOS HÁBITOS

ALIMENTARES DE

ESCOLARES
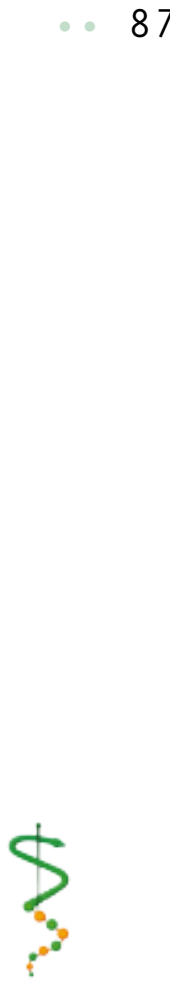

REV, ODONTOL.

Univ. CID. São

PAULO

2016; 28(2):

$86-95$, MA I - AGO 
TAVARES RNM

TEIXEIRA ALH

COSTA LL

AMORIM LCT

LICATTI LCM

ZANIN $L$

FLORIO FM

OLIVEIRA

AMGPREVALÊNCIA

DE CÁRIE

ASSOCIADA À

INFLUÊNCIA

DA PROPAGANDA

NOS HÁBITOS

ALIMENTARES DE

ESCOLARES

\section{8}

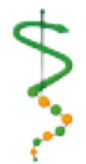

REV, ODONTOL.

UNIV. CID, SÃO

PAULO

2016; 28(2):

$86-95$, MA I - AG 0
I SSN 1983-5183

cação.

A responsabilidade compartilhada entre sociedade, escola, setor produtivo e setor público é um desafio e o caminho para a construção de modos de vida pautados também nos princípios da promoção da saúde e da prevenção de doenças, sendo importante as intervenções governamentais que visem proteger a criança de riscos, compreendidas como instrumentos para a garantia do direito a uma alimentação adequada e saudável $7,8,9,10,11,12,13$.

Nesse contexto, dada a pouca publicação acerca da associação entre cárie dentária e propagandas televisivas de alimentos no Brasil, este estudo objetivou avaliar a influência dos comerciais de televisão nos hábitos alimentares de adolescentes e a sua associação com a prevalência de cárie.

\section{MÉTODOS}

Estudo epidemiológico observacional transversal, desenvolvido no município de Itatiba, região metropolitana de Campinas, São Paulo, com população estimada em 104.822 habitantes. Os indicadores sociais referentes ao município em relação à qualidade de vida da população apresentam um Índice de Desenvolvimento Humano (IDH) de 0,828. A população do estudo foi constituída por 313 adolescentes com idade de 12 anos, ambos os gêneros, matriculados no sétimo ano do Ensino Fundamental II de escolas da rede pública do município de Itatiba, São Paulo. Com base em estudo prévio ${ }^{14}$, para o cálculo do tamanho da amostra foi utilizado o valor da média (CPO-D=2.5) e desvio-padrão de 1.8, Índice de Confiança de 95\%, precisão de $10 \%$ e efeito do desenho (deff) de 2 . Foram somados $20 \%$ ao total, a fim de compensar eventuais perdas de elementos da amostra, que resultou em 341. Foram excluídos da pesquisa alunos cujos pais não assinaram o TCLE; aqueles que se recusaram a responder ao questionário ou o fizeram de forma incorreta; aqueles que não se submeteram ao exame clínico e aqueles com necessidades especiais de saúde, resultando em uma amostra final de 294 estudantes.

O estudo foi realizado no período de agosto a outubro de 2013. Na primeira etapa, examinadores calibrados (Kappa = $0,91)$ realizaram exame epidemiológico, utilizando-se o índice CPO-D com o objetivo de se verificar a história de cárie. Para a calibração foram seguidos os critérios descritos no manual de calibração de examinadores da Organização Mundial da Saúde de $2003^{15}$.

Paralelamente, aplicou-se um questionário estruturado validado do estudo de Ghimire e $\operatorname{Rao}^{8}$ (2013), para avaliar a associação da influência da propaganda televisiva na prevalência da cárie. $\mathrm{O}$ questionário contemplou dez perguntas sobre os hábitos de assistir televisão e de alimentação, e uma sobre o canal de TV preferido. Admitiu-se mais de uma resposta por aluno em cada questão.

Na segunda etapa, os pesquisadores assistiram por sete dias consecutivos, durante o horário mais citado, a programação das duas emissoras mais assistidas pelos alunos, segundo respostas do questionário aplicado. As propagandas foram quantificadas, descritas e classificadas em: propagandas de produtos não alimentícios, de produtos alimentícios e propagandas de produtos de higiene. Para as propagandas de produtos não alimentícios foram incluídos produtos como brinquedos, medicamentos, automóveis, dentre outros. As propagandas de produtos alimentícios foram divididas em propagandas de produtos cariogênicos e não cariogênicos. As propagandas classificadas como "produtos cariogênicos" contemplaram alimentos industrializados que tinham em sua composição o açúcar refinado como, por exemplo, chocolates, bebidas açucaradas, biscoitos, entre outros; e aquelas de "produtos não cariogênicos" se referiam aos alimentos sem açúcar refinado, como frutas, legumes, verduras, carnes, entre outros. As propagandas de produtos de higiene contemplaram produtos de higiene corporal como sabonete, shampoo, cotonetes, sabonete íntimo etc. e produtos de higiene bucal como fio dental, escova de dente, pasta de dente, enxaguatório bucal, etc.

Para fins da análise estatística o índice CPOD foi dicotomizado na mediana $(\mathrm{CPOD}=0 ; \mathrm{CPOD}>0)$. A seguir as variáveis previamente selecionadas por análises in- 
dividuais pelos testes de Qui-quadrado e Exato de Fisher foram testadas em um modelo de regressão logística múltipla, permanecendo no modelo as variáveis com $\mathrm{p} \leq 0,10$. Para isso foi utilizado o nível de significância de $10 \%$, a fim de não se perder informações importantes por se tratar de estudo de prevenção. Pela regressão logística múltipla foram estimados os odds ratio ajustados e os respectivos intervalos de confiança de 95\%. Todas as análises foram realizadas no programa SAS (Institute Inc., Cary, NC, USA, Release 9.2, 2010). Adicionou-se, posteriormente, a correlação de Spearman para testar a associação da prevalência de cárie com o tempo de permanência em frente à televisão, para a qual o CPOD foi categorizado de acordo com a prevalência de cárie e o tempo dicotomizado em $>90$ min e $<90$ min. Para se avaliar se houve correlação foi utilizado o programa Bioestat 5.0.

Este estudo foi aprovado pelo Comitê de Ética em Pesquisa da Faculdade São Leopoldo Mandic (Protocolo: 428369).

\section{RESULTADOS}

Observou-se que $52.38 \%$ dos participantes eram do sexo feminino e $48.62 \%$ do masculino. 288 (97.95\%) escolares assistiam TV e, desse percentual, 171 $(59,37 \%)$ assistiam à programação no horário de 18 h às $20 \mathrm{~h} ; 84(29,16 \%)$ as- sistiam TV das 9 às 12h; $70(24,30 \%)$ das 12 às 16h; 61 (21,18\%) das 16 às 18h; e 8 (2,77\%) não informaram o horário em que assistiam TV. Com relação ao tempo de permanência em frente à TV, observou-se que $34(11,80 \%)$ escolares assistiam TV por tempo inferior a 30 minutos; 106 $(36,80 \%)$ assistiam de 60 a 90 minutos; $138(47,91 \%)$ por mais de 90 minutos e $10(3,47 \%)$ não responderam.

$\mathrm{Na}$ maioria, os entrevistados 159 $(55,20 \%)$ relataram assistir às emissoras de TV GLOBO $(28,47 \%, n=82)$ e SBT $(26,73 \%, \mathrm{n}=77)$. Os outros canais mencionados representaram $44,80 \%$ de acesso, distribuídos em 18 emissoras, entre elas a BAND $(4 \%, n=12)$. Os resultados mostraram que os maiores percentuais de escolares com cárie assistiam às emissoras SBT $(p=0.05)$ e BAND $(p=0.06)$, relacionados ao horário das $9 \mathrm{~h}$ às $12 \mathrm{~h}(\mathrm{p}=0.01)$. A comparação foi feita entre os que assistiam e os que não assistiam a cada emissora, devido à duplicidade nas respostas. Entre os que assistem à Globo, 36,5\% das crianças apresentaram CPOD $>0$ e entre os que não assistem $38.0 \%$, apresentando uma diferença de 1,5\% (38-36.5). Para a Band essa diferença foi de $3,6 \%$ e para o SBT foi $12,7 \%$ (Tabela 1 ).

Os estudantes que assistiam ao canal SBT e os que assistiam TV das 9 às 12 horas apresentaram 1,73 vezes

Tabela 1. Análises individuais das associações das variáveis analisadas com o CPOD.

\begin{tabular}{|c|c|c|c|c|c|c|c|c|}
\hline \multirow[t]{2}{*}{ Variável } & & \multirow{2}{*}{$\begin{array}{c}\mathrm{CPOD}=0 \\
\mathrm{~N}\end{array}$} & \multicolumn{3}{|c|}{ CPO̊D $>0$} & \multirow{2}{*}{$\begin{array}{c}\text { Odds-ratio } \\
\text { bruto }\end{array}$} & \multirow[t]{2}{*}{ IC95\% } & \multirow[t]{2}{*}{ p-valo } \\
\hline & & & $\%$ & $\mathrm{~N}$ & $\%$ & & & \\
\hline Idade & 12 anos & 182 & 62,32 & 110 & 37,6 & 1,00 & & 0,3681 \\
\hline \multirow[t]{2}{*}{ Sexo } & Feminino & 96 & 62,3 & 58 & 37,7 & 1,00 & & 0,8726 \\
\hline & Masculino & 86 & 61,4 & 54 & 38,6 & 1,04 & $0,65-1,66$ & \\
\hline \multirow[t]{2}{*}{ Assiste TV } & Não & 5 & 83,3 & 1 & 16,7 & 1,00 & & 0,4130 \\
\hline & Sim & 177 & 61,5 & 111 & 38,5 & 3,14 & $0,36-27,19$ & \\
\hline \multirow[t]{8}{*}{ Horário } & 9 as $12 \mathrm{hs}$ & 44 & 51,8 & 41 & 48,2 & 1,86 & $1,11-3,12$ & 0,0175 \\
\hline & Não & 134 & 66,7 & 67 & 33,3 & 1,00 & & \\
\hline & 12 as $16 \mathrm{hs}$ & 46 & 64,8 & 25 & 35,2 & 0,86 & $0,49-1,51$ & 0,6091 \\
\hline & Não & 132 & 61,4 & 83 & 38,6 & 1,00 & & \\
\hline & 16 as $18 \mathrm{hs}$ & 38 & 61,3 & 24 & 38,7 & 1,05 & $0,59-1,88$ & 0,8620 \\
\hline & Não & 140 & 62,5 & 84 & 37,5 & 1,00 & & \\
\hline & 18 as $20 \mathrm{hs}$ & 112 & 65,1 & 60 & 34,9 & 0,74 & $0,45-1,20$ & 0,2174 \\
\hline & Não & 66 & 57,9 & 48 & 42,1 & & & \\
\hline
\end{tabular}

TAVARES RNM

TEIXEIRA ALH

COSTA LL

AMORIM LCT

LICATTI LCM

ZANIN L

FLORIO FM

OLIVEIRA AMG

PREVALENCIA DE

CÁRIE ASSOCIADA

À INFLUÊNCIA

DA PROPAGANDA

NOS HÁBITOS

ALIMENTARES DE

ESCOLARES

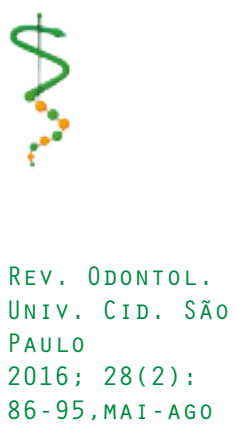




\begin{tabular}{|c|c|c|c|c|c|c|c|c|c|}
\hline \multirow{2}{*}{$\begin{array}{r}\text { TAVARES RNM } \\
\text { TEIXEIRA ALH } \\
\text { COSTA LL }\end{array}$} & \multicolumn{2}{|l|}{ Variável } & \multirow{2}{*}{$\begin{array}{c}\text { CPOD }=0 \\
20\end{array}$} & \multicolumn{3}{|c|}{$\mathrm{CPOD}>0$} & \multirow{2}{*}{$\frac{\text { Odds-ratio }}{1,00}$} & \multirow[t]{2}{*}{ IC95\% } & \multirow{2}{*}{$\frac{p \text {-valor }}{0,4223}$} \\
\hline & Minutos & Menos de 30 & & 55,6 & 16 & 44,4 & & & \\
\hline AMORIM LCT & & 60 a 90 & 63 & 59,4 & 43 & 40,6 & 0,85 & $0,40-1,83$ & \\
\hline $\begin{array}{r}\text { LICATTI LCM } \\
\text { ZANIN } L\end{array}$ & & Mais de 90 & 90 & 65,7 & 47 & 34,3 & 0,65 & $0,31-1,38$ & \\
\hline FLORIO FM & \multirow[t]{2}{*}{ Globo } & Não & 11 & 62,0 & 68 & 38,0 & 1,00 & & 0,8224 \\
\hline OLIVEIRA & & Sim & 47 & 63,5 & 27 & 36,5 & 0,94 & $0,54-1,64$ & \\
\hline $\begin{array}{r}M G P R E V A L \hat{E} N C I A \\
D E \quad C \bar{A} R I E\end{array}$ & \multirow[t]{2}{*}{ SBT } & Não & 115 & 66,5 & 58 & 33,5 & 1,00 & & 0,0520 \\
\hline ASSOCIADA & & Sim & 43 & 53,8 & 37 & 46,2 & 1,70 & $0,99-2,93$ & \\
\hline INFLUÊECIA & \multirow[t]{2}{*}{ Band } & Não & 154 & 63,9 & 87 & 63,1 & 1,00 & & 0,0619 \\
\hline NOS HÁBITOS & & Sim & 4 & 33,3 & 8 & 66,7 & 3,54 & $1,04-12,09$ & \\
\hline \multirow{12}{*}{$\begin{array}{r}\text { LIMENTARES DE } \\
\text { ESCOLARES }\end{array}$} & \multirow[t]{2}{*}{ Cartoon } & Não & 148 & 62,4 & 89 & 37,6 & 1,00 & & 0,9966 \\
\hline & & Sim & 10 & 62,5 & 6 & 37,5 & 1,00 & $0,35-2,84$ & \\
\hline & \multirow[t]{2}{*}{ Disney } & Não & 131 & 59,8 & 88 & 40,2 & 2,59 & $1,08-6,21$ & 0,0282 \\
\hline & & Sim & 27 & 79,4 & 7 & 20,6 & 1,00 & & \\
\hline & Comerciais & Não & 54 & 68,8 & 28 & 34,2 & 1,00 & & 0,3568 \\
\hline & Compra & Não & 64 & 59,3 & 44 & 40,7 & 0,80 & $0,49-1,32$ & 0,3853 \\
\hline & produtos & Sim & 107 & $\begin{array}{l}64.5 \\
64,5\end{array}$ & 59 & 35,5 & 1,00 & & \\
\hline & Alimentos & Não & 65 & 62,5 & 39 & 37,5 & 1,00 & & 0,8827 \\
\hline & & Sim & 51 & 61,4 & 32 & 38,6 & 1,04 & $0,58-1,89$ & \\
\hline & $\begin{array}{l}\text { Refrige- } \\
\text { rante }\end{array}$ & Não & 90 & 62,9 & 53 & 37,1 & 1,00 & & 0,6457 \\
\hline & & Sim & 26 & 59,1 & 18 & 40,9 & 1,18 & $0,59-2,34$ & \\
\hline & Fio dental & Não & 105 & 61,8 & 65 & 38,2 & 1,00 & & 0,8117 \\
\hline \multirow[t]{3}{*}{$\therefore 90 \ldots$} & & Sim & 11 & 64,7 & 6 & 35,3 & 0,88 & $0,31-2,50$ & \\
\hline & \multirow[t]{2}{*}{$\begin{array}{l}\text { Enxaguató } \\
\text { rio }\end{array}$} & Não & 98 & 61,6 & 61 & 38,4 & 1,00 & & 0,7899 \\
\hline & & Sim & 18 & 64,3 & 10 & 35,7 & 0,89 & $0,39-2,06$ & \\
\hline
\end{tabular}

Fonte : Autoria própria

Tabela 2. Análise de regressão logística múltipla das variáveis analisadas associadas como CPOD.

\begin{tabular}{ccccccccc}
\hline \hline Variável & & CPOD $=0$ & & CPOD $>0$ & & Odds-ratio & IC95\% & p-valor \\
\hline \multirow{2}{*}{ SBT } & & $\mathrm{N}$ & $\%$ & $\mathrm{~N}$ & $\%$ & ajustado & & \\
& Não & 115 & 66,5 & 58 & 33,5 & 1,00 & & 0,0506 \\
\multirow{2}{*}{ Horário } & Sim & 43 & 53,8 & 37 & 46,2 & 1,73 & $1,00-3,02$ & 0,0770 \\
& 9 & 44 & 51,8 & 41 & 48,2 & 1,66 & $0,95-2,90$ & \\
& Não & 134 & 66,7 & 67 & 33,3 & 1,00 & & \\
\hline \hline
\end{tabular}

Fonte : Autoria própria

Fonte : Autoria própria

(IC95\%:1.00-3.02; $p=0,0506)$ e 1,66 vezes (IC95\%:0,95-2,90; $\mathrm{p}=0,0770)$ respectivamente mais chance de ter $\mathrm{CPOD}>0$ (Tabela 2).

Durante sete dias foram veiculados um total de 303 propagandas nas duas emissoras mais assistidas (GLOBO e SBT). Dessas propagandas, $22,11 \%$ eram de produ-

tos alimentícios distribuídos em 15,51\% de alimentos cariogênicos e $6,60 \%$ de alimentos não cariogênicos; 5,94\% de produtos de higiene corporal, ressaltando-se que nenhuma propaganda de produtos de higiene oral foi apresentada nesse período; e 71,95\% de outros produtos (brinquedos, automóveis, roupas, serviços 
Tabela 3. Percentual de escolares por produtos veiculados nas propagandas de TV com potencial de influência para a compra.

\begin{tabular}{ll}
\hline \hline PRODUTOS & ESCOLARES $(\%)$ \\
\hline ALIMENTOS & 40,00 \\
REFRIGERANTES & 24,13 \\
ENXAGUATÓRIO BUCAL & 13,10 \\
FIO DENTAL & 11,72 \\
SABONETE & 7,59 \\
ROUPAS & 46,20 \\
BICICLETAS & 11,72 \\
BRINQUEDOS & 11,30 \\
OUTROS & 20,68 \\
\hline \hline
\end{tabular}

Fonte : Autoria própria.

bancários, etc).

Foi registrado que, dos 207 estudantes que assistiam aos comerciais nos intervalos das programações, 70,05\% $(n=145)$ relataram sentir-se influenciados pelas propagandas televisivas e compravam ou pediam para os pais comprarem produtos vistos nos comerciais televisivos. A Tabela 4 mostra a frequência de escolares, em função do provável consumo por sugestão das propagandas.

A análise de correlação foi positiva entre a prevalência de cárie e o tempo de exposição à televisão ( $r s=0,92 ; p=0,02)$ (Tabela 4).

\section{I SCUSSÃO}

As propagandas televisivas têm forte influência na escolha de consumo de seus telespectadores, interferindo nos hábitos comportamentais e alimentares, principalmente em crianças e adolescentes, sendo a propaganda um determinante importante de consumo alimentar ${ }^{16,17}$. Dessa forma, é relevante o cuidado com o que as crianças assistem nas programações televisivas, dando-se ênfase ao papel mediador dos pais nesse processo de influência, pois parece existir uma correlação entre os alimentos anunciados nos programas televisionados com os pedidos de compras de alimentos feitos pelas crianças ${ }^{17}$

Motta-Gallo et al. ${ }^{18}$ (2013) identificaram a influência das propagandas de alimentos veiculadas pela televisão nos hábitos de consumo alimentar das crianças, quando pais relataram que a decisão de compra da família passou a ser regulada pelas solicitações das crianças motivadas pelas propagandas televisivas. Em decorrência dessas observações, os resultados do estudo mostraram que o tempo de permanência em frente à TV passou a ser também levado em consideração, pois favorece maior exposição por parte do telespectador às mensagens de marketing.

Quando se avalia o tempo de exposição às propagandas televisivas, os resultados apontam para uma maior prevalência de cárie nas crianças que passavam mais tempo assistindo televisão (> 90 minutos), de forma a se inferir haver uma correlação positiva ( $r s=0.92$ ) entre tempo de exposição à propaganda e prevalência de cárie. O presente achado está de acordo com estudos prévios ${ }^{11,19,20}$. Em uma das pes-

Tabela 4. Correlação da prevalência de cárie com o tempo de exposição à TV.

\begin{tabular}{lccccc}
\hline \hline Tempo & \multicolumn{3}{c}{ Prevalência de cárie } \\
\hline & Muito baixa & Baixa & Média & Alta & Muito alta \\
$>90$ min & 23 & 13 & 4 & 1 & 1 \\
$<90$ min & 33 & 8 & 2 & 2 & 0 \\
Coeficiente de Correlação de Spearman & & & rs $=0,92$ & p-valor $=0,02$ \\
\hline \hline
\end{tabular}

TAVARES RNM

TEIXEIRA ALH

COSTA LL

AMORIM LCT

LICATTI LCM

ZANIN L

FLORIO FM

OLIVEIRA AMG

PREVALENCIA DE

CÁRIE ASSOCIADA

À INFLUENCIA

DA PROPAGANDA

NOS HÁBITOS

ALIMENTARES DE

ESCOLARES 
TAVARES RNM

TEIXEIRA ALH

COSTA LL

AMORIM LCT

LICATTI LCM

ZANIN $L$

FLORIO FM

OLIVEIRA

AMGPREVALÊNCIA

DE CÁRIE

ASSOCIADA À

INFLUENNCIA

DA PROPAGANDA

NOS HÁBITOS

ALIMENTARES DE

ESCOLARES
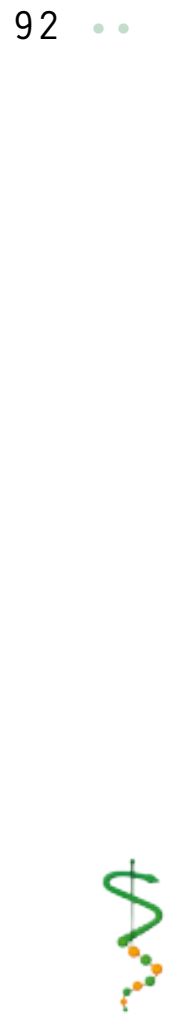

REV. ODONTOL. UNIV. CID. SÃO

PAULO

2016; 28(2):

$86-95$, MAI - AGO quisas citadas, crianças que permaneceram em frente à TV por um período igual ou superior a três horas tiveram consumo maior de alimentos não saudáveis e, portanto, maior risco à cárie ${ }^{11}$. Ainda de acordo com os mesmos autores, os alimentos não saudáveis são mais anunciados durante a programação infantil, o que poderia justificar o fato de que $48.2 \%$ dos 81 escolares que assistiam à TV no horário das 9 às 12, horário dedicado ao público infantil, apresentaram CPOD $>0(p=0.01)$. Nessa pesquisa, o horário indicado como o mais assistido pelos escolares, foi das 18 às 20 horas, não apresentando significância nos resultados.

No presente estudo, o percentual de comerciais de alimentos e bebidas encontrado foi de $22,11 \%$ do total avaliado, resultado semelhante ao de outros autores que relataram que os produtos alimentícios representam cerca de $20 \%$ da mídia em geral 3,5,8. Entretanto, obervou-se que dentre as propagandas de alimentos e bebidas veiculadas, $15,51 \%$ eram de alimentos considerados cariogênicos e possuíam forte apelo de marketing para a criança. Esse dado se tornou relevante, considerando-se que adolescentes ainda não possuem senso crítico para se posicionarem diante das propagandas ${ }^{21,22}$. Resultados semelhantes foram encontrados por Pimenta et al. ${ }^{3}$ (2011), ao analisarem 2.167 comerciais, dos quais 542 eram de bebidas e alimentos. Os autores concluíram que a maioria era de baixa qualidade nutricional e rica em açúcares; o refrigerante foi o mais presente nas propagandas; e a frequência desses comerciais era maior nos finais de semana.

Em relação aos canais assistidos, a maior porcentagem de escolares com CPOD $>0$ foi observada entre os que assistiam os canais abertos SBT $(p=0.0520)$ e Band $(0,0619)$, com menor porcentagem encontrada entre os que assistiam Disney Chanel $(p=0,0282)$. Esses resultados são reforçados pelos achados de Mainardi et al. 23 (1998), que demonstraram que o SBT foi a segunda emissora de canal aberto com maior número de comerciais de influência negativa (alimentos cariogênicos) dirigidos para o público infantil. O fato de os pesquisadores terem assistido ape- nas às programações das emissoras SBT e Globo deveu-se ao delineamento da pesquisa, que considerou apenas os canais mais assistidos pelos escolares, apontando para o SBT a maior associação de cárie (CPOD $>0$ ).

Em relação aos produtos escolhidos pelos escolares influenciados pelas propagandas nesta pesquisa, os alimentos $(40 \%)$ e refrigerantes $(24,13 \%)$ foram os mais indicados, o que é corroborado pelos resultados de Ghimire e $\operatorname{Rao}^{8}$ (2013) que, ao avaliarem a influência das propagandas sobre crianças e seu impacto sobre a saúde oral, observaram que, de $66 \%$ das crianças que pediam para os pais comprarem alimentos veiculados nos comerciais, 48,5\% referiam os refrigerantes e a maioria possuía índices de cárie elevado, o que demonstrou uma forte evidência da influência da televisão na alimentação infantil. Esse fato deve ser tratado não só como uma questão de saúde, mas também como uma questão de educação, uma vez que a mídia é formadora de opiniões. Os meios de comunicação de massa induzem às práticas alimentares erradas pelas crianças e estas tendem a solidificar-se na vida adulta ${ }^{7}$.

Diante da polêmica sobre crianças e adolescentes serem alvos de anúncios publicitários, alguns países da Europa já proibiram propagandas voltadas para esse público ${ }^{21}$. No Brasil, a Resolução № 163 do Conselho Nacional dos Direitos da Criança e do Adolescente (CONANDA), publicada no Diário Oficial da União em 13/03/2014, considera abusiva a prática de direcionamento da publicidade e de comunicação mercadológica à criança com a intenção de persuadi-la para o consumo de qualquer produto ou serviço. As empresas passaram a redirecionar ao público adulto (pais) toda a comunicação mercadológica que hoje tem a criança como meta, tornando assim o horário mais assistido por adultos alvo de propagandas diversas, principalmente de alimentos não saudáveis como os ricos em açúcares e gorduras, fato comprovado por nossa pesquisa, que apresentou nos resultados um percentual de 15,51\% de propagandas de alimentos cariogênicos veiculadas no horário das 
18 às 20 horas, sendo este o mais assistido pelos escolares de acordo com os dados levantados na pesquisa.

A alimentação assume um contexto social e papel central nas interações sociais, permitindo a participação de normas na condução do comportamento alimentar, sendo capaz de trazer o julgamento social e transmitir informações dos indivíduos por meio dos fenômenos de facilitação social e gerenciamento de impressão ${ }^{24}$.

Nesse contexto, o público infantil e adolescente, por sua fragilidade no processamento de informações publicitárias, está altamente vulnerável à aquisição de hábitos inadequados de alimentação e de vida, devendo-se, portanto, alertar pais e responsáveis quanto aos riscos de uma alimentação não saudável; para isso a escola deveria funcionar como um espaço integrado à família, para reforçar a necessidade de um cardápio variado que contemplasse os nutrientes vitais para o adequado desenvolvimento da criança, de forma que a alimentação saudável aprendida nas atividades pedagógicas também seja aplicada extramuros e que as crianças se tornem agentes promotores da própria saúde e de sua família.

É importante que se evidencie algumas limitações nesse estudo. A pesquisa foi conduzida seguindo a metodologia proposta por Ghimire e $\operatorname{Rao}^{8}$ (2013), em que a análise das propagandas era feita no horário indicado como o mais utilizado pela maioria dos componentes da amostra; en- tretanto, esse horário apontado pelos escolares da amostra nesta pesquisa, $18 \mathrm{~h}$ às $20 \mathrm{~h}$, não correspondeu ao maior número de crianças com CPOD $>0$, estas foram mais frequentes no horário de $9 \mathrm{~h}$ às $12 \mathrm{~h}$ que não recebeu análise das propagandas veiculadas, porém, horário destinado a programações infantis. A autoaplicação do questionário pode ter dificultado a sua compreensão pelas crianças.

Este estudo aponta para fatores importantes que podem influenciar a ingestão de alimentos industrializados não saudáveis por crianças e adolescentes. Mais pesquisas investigando as questões inerentes à alimentação em escolares devem ser incentivadas, principalmente após a resolução $N^{o} 163$ do CONANDA de $13 / 3 / 2014$, pois, ainda que se redirecione as propagandas para o horário apropriado ao público adulto, estas também são vistas por crianças e adolescentes, fato comprovado pela presente pesquisa. Estudos que têm avaliado associações de propagandas de alimentos na televisão e a prevalência de cárie dentária em escolares ainda são escassos.

\section{CONCLUSÃO}

As propagandas veiculadas na televisão e o tempo de exposição em frente à TV podem influenciar os hábitos alimentares de crianças e adolescentes de forma negativa, levando ao consumo de alimentos ricos em açúcares, considerados cariogênicos e de pouco valor nutritivo.
TAVARES RNM

TEIXEIRA ALH

COSTA LL

AMORIM LCT

LICATTI LCM

ZANIN L

FLORIO FM

OLIVEIRA AMG

PREVALENCIA DE

CÁRIE ASSOCIADA

À INFLUÊNCIA

DA PROPAGANDA

NOS HÁBITOS

ALIMENTARES DE

ESCOLARES

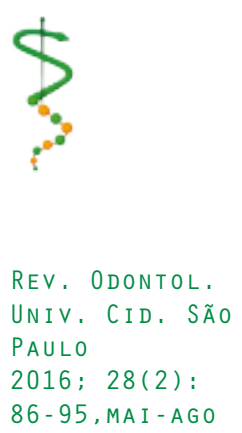


TAVARES RNM

TEIXEIRA ALH

COSTA LL

AMORIM LCT

LICATTI LCM

ZANIN $L$

FLORIO FM

OLIVEIRA

AMGPREVALÊNCIA

DE CÁRIE

ASSOCIADA À

INFLUENNCIA

DA PROPAGANDA

NOS HÁBITOS

ALIMENTARES DE

ESCOLARES

94

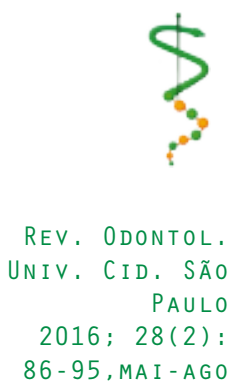

\section{REFERÊNCIAS}

1. Thylstrup A, Fejerskov O. Tratado de cariologia. São Paulo: Cultura Médica; 1988.

2. Fejerskov O, Kidd E. Cárie dentária: a doença e seu tratamento clínico. 2. ed. São Paulo: Santos; 2013.

3. Pimenta DV, Masson DF, Bueno MB. Análise das propagandas de alimentos veiculadas na televisão durante a programação voltada ao público infantil. J Health Sci Inst 2011 29(1):52-5.

4. Collaço JHL. Restaurantes de comida rápida, os fast-foods, em praças de alimentação de shopping centers: transformações no comer. Estudos Históricos 2004 1(33):116-35.

5. Almeida SS, Nascimento PCBD, Quaioti TCB. Quantidade e qualidade de produtos alimentícios anunciados na televisão brasileira. Rev Saúde Pública 2002 jun;36(3):353-5.

6. Fechine ÁDL, Machado MMT, Lindsay AC, Fechine VAL, Arruda CAM. Percepção de pais e professores sobre a influência dos alimentos industrializados na saúde infantil. Rev Bras Promoç Saúde, Fortaleza 2015 jan/ mar;28(1):16-22.

7. Henriques P, Sally EO, Burlandy L, Beiler RM. Regulamentação da propaganda de alimentos infantis como estratégia para a promoção da saúde. Ciênc saúde coletiva 2012 fev;17(2):481-90.

8. Ghimire N, Rao A. Comparative evaluation of the influence of television advertisements on children and caries prevalence. Glob Health Action 2013 6(20066.

9. Fogaça LMP, Oliveira DS, Oliveira T. Análises das propagandas alimentícias e sua influência na obesidade infantil. Interciencia \& Sociedade 2014 3(2):916.
PC, Oliveira TP. A saúde pública e a regulamentação da publicidade de alimentos. Brasília: Ministério da Saúde 2009 [Acesso em]; Disponível em: http://189.28.128.100/nutricao/docs/ geral/regulamentaPublicidadeAlimentos.pdf.

11. Bibiano CRD, Schmidt IT. A influencia da televisão na alimentação infantil: uma questão educativa. Colloquium Humanarum 2010 7(2):74-9.

12. Zeng X, Sheiham A, Sabbah W. The association between dental caries and television viewing among Chinese adolescents in Guangxi, China. BMC Oral Health 2014 14(138.

13. Murray R, Bhatia J, Okamoto J, Allison M, Ancona R, Attisha E, et al. Snacks, Sweetened Beverages, Added Sugars, and Schools. Pediatrics 2015 135(3):575-83.

14. Santos SL, Batalha MO. Propaganda de alimentos na televisão: uma ameaça à saúde do consumidor. Rev Adm 2010 dez;45(4):373-82.

15. Brasil. Ministério da Saúde. Departamento de Atenção Básica. Projeto SB Brasil 2003: condições de saúde bucal da população brasileira 2002-2003: resultados principais. Brasília: Ministério da Saúde; 2004.

16. Mattos MC, Nascimento PCBD, Almeida SS, Costa TMB. Influência de propagandas de alimentos nas escoIhas alimentares de crianças e adolescentes. Psicol teor prat 2010 mar ;12(3):34-51.

17. Andrade A, Bosi MLM. Mídia e subjetividade: impacto no comportamento alimentar feminino. Rev Nutr 2003 jan;16(1):117-25.

18. Motta-Gallo S, Gallo P, Cuenca A. Influência da televisão nos hábitos alimentares de crianças do nordeste brasileiro. Rev bras crescimento desenvolv hum 2013 23(1):87-93. 
19. Ruano Ruano I, Serra Pujol ME. Hábitos de vida en una población escolar de Mataró (Barcelona) asociados al número de veces diarias que ve televisión y al consumo de azúcares. Rev Esp Salud Pública, Madrid 1997 71(5):487-98.

20. Barr-Anderson DJ, Larson NI, Nelson MC, Neumark-Sztainer D, Story M. Does television viewing predict dietary intake five years later in high school students and young adults? Int J Behav Nutr Phys Act 2009 6(7.

21. Sampaio ISV. Televisão, publicidade e infância. 2.ed. ed. São Paulo: Annablume; 2004.
22. Linn S. Crianças do consumo: a infância roubada. São Paulo: Instituto Alana; 2006 [Acesso em]; Disponível em: http://www.cvdee.org.br/evangelize/ pdf/1_0743.pdf.

23. Mainardi APR, Oliveira MDM, Finger AT, Pinto AS, Leonardelli A, Dariva $C$, et al. Influência dos comerciais da televisão nos hábitos dietéticos. RFO, Passo Fundo 1998 jan-jun;3(1):35-40.

24. Batista MT, Lima ML. Comer o quê com quem?: Influência social indirecta no comportamento alimentar ambivalente. Psicol Reflex Crit 2013 26(1):113-21.

Recebido em 11/05/2016

Aceito em 08/08/2016
TAVARES RNM

TEIXEIRA ALH

costa Ll

AMORIM LCT

LICATTI LCM

ZANIN L

FLORIO FM

OLIVEIRA AMG

PREVALENCIA DE

CÁRIE ASSOCIADA

à INFLUÊNCIA

DA PROPAGANDA

NOS HÁBITOS

ALIMENTARES DE

ESCOLARES

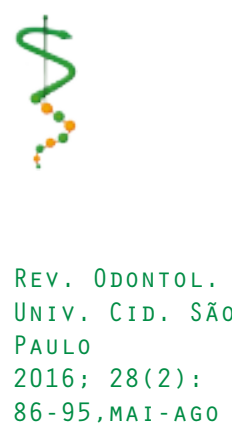

